\title{
CODE TO CRAFT - BEYOND THE VOXEL
}

\author{
JEROME FRUMAR \\ Spatial Information Architecture Laboratory \\ School of Architecture and Design \\ Royal Melbourne Institute of Technology University \\ GPO Box 2476V, Melbourne, VIC 3001, Australia \\ jerome.frumar@rmit.edu.au
}

\begin{abstract}
The digital nature of post-industrial societies has profound implications for architectural design, documentation and construction. Digital tools and technologies bridge the representational divide between conception and realization, empowering architects to regain control of the design, fabrication and assemblage processes. This paper will discuss ideas and concepts to facilitate the fabrication of non-standard, context-specific, geometrically complex architecture and components. Two case studies exploring digital fabrication and metal casting will be described alongside implications for the fields of architectural design and construction.
\end{abstract}

Keywords: architecture, craft, craftsmanship, component manufacturing, computer aided manufacturing, digital design, jewelry, metal casting, rapid prototyping.

\section{Introduction}

Digital tools are prevalent in many aspects of contemporary global culture, streamlining and consolidating multifaceted systems and automating repetitious tasks. They are increasingly instrumental for developing complex and holistic solutions to complicated problems. These technologies have the potential to extend human knowledge and the 'vocabulary' we use to engage and express ourselves.

The advent of CAD (computer-aided design) and CAM (computer-aided manufacturing) has transformed the paradigm traditionally associated with design, fabrication and construction. CAD/CAM tools enable the direct fabrication of a virtual 3D model using a diverse range of materials and techniques. As such, these technologies bridge a representational divide between conception, design and realization. Highly integrated in the fields of aerospace, automotive, industrial and nautical design, the use of CAD/CAM has only recently prompted interest from the building industry and allied fields of architecture and engineering. These professions are poised for change as they become empowered through a nascent medium where representation is synonymous with the information needed for data-driven fabrication.

The integration of digital design tools in teaching, learning and practice over the past decade has rendered CAD obligatory as a drafting aid in much of the architecture and engineering industries. Recently, increased computing power has enabled CAD tools to mature and suggest possibilities far beyond their origins. Those at the forefront of research-based architecture 
practice have recognized the potentials of appropriating an array of computation and fabrication strategies to extend design, manufacturing and construction capabilities.

Complex forms are increasingly frequent in the contemporary architectural lexicon and these necessitate novel and innovative means of communication. Concurrently, commercially available and emerging CAM technologies facilitate new geometric potentials and bypass the need for traditional 2D drawings to specify $3 \mathrm{D}$ relationships, dimensions and other vital fabrication information. This digital convergence of representation and production embodies one of the most important opportunities and challenges facing the contemporary architecture industry.

\section{Beyond Aggregates}

As data driven CAM technologies increase in scale they can be used to fabricate components suitable for building and construction. Schodek outlines a host of architects and projects that have made headway into automated fabrication and rapid construction techniques for geometrically complex non-standard architecture. It is interesting to observe however, that the majority of work thus far considered in these terms has been surface-oriented with structural systems manifest as sampled 2.5D instances of a particular 3D 'master geometry'. Achieving a precise (re)production of complex digital form has consistently posed fabrication challenges. Generally, so-called smooth or non-planar geometries have been accomplished with a combination of CNC (Computer Numerically Controlled) milling and casting/forming (glass, composites, plastics, concrete, metal etc) but have remained in the domain of surface and/or surface treatment. Bernhard Franken's BMW Deutschland Pavilion built for the Frankfurt motor show in 1999 exhibits two of the dominant approaches to the construction of non-standard architecture. 305 doubly curved cast Plexiglas panels enclose an aggregate system of interlocking planar sections that define the structure and interior. In his essay Blurring the Lines, Andre Chaszar queries a pivotal characteristic of contemporary and emerging architectural design, fabrication and construction;

"Will CAM eventually be able to produce fully 3D forms in building scale elements or will complex forms continue to be made from 2D elements assembled into 3D aggregates?" (14)

This is an important question. It suggests thinking beyond surface to increasingly efficient and context-specific systems that suitably compliment digitally generated architectural forms. In an interview with Pyotr Kudryavstev, Franken acknowledges this necessity stating that many contemporary architects "are interested mostly in the external characteristics of a building, its surface ... These days, architecture is seen merely as designing a sort of clothing for buildings." Currently, industrial processes for realizing "fully 3D" complex objects fall within the broad genres of additive and subtractive fabrication. These tools represent the framework for an almost limitless palette of geometries and support the quest to unify surface, structure and ornament. Due to the ongoing development of new materials and larger scale fabrication platforms they are increasingly applicable in the fabrication of non-standard architecture and components. An emerging avenue of particular interest is rapid manufacturing, the direct fabrication of functional end-use parts from 3D model data. Viable at the scale of hardware and industrial design, the building industry awaits the development of sufficiently large platforms where freeform processes such as laser sintering or layer deposition can be used to directly fabricate full-scale building components. An example is Concrete Contour Crafting, a megascale construction technique being developed by Behrokh Khoshnevis at the Department of Industrial and Systems Engineering, University of Southern California. Using a layer deposition method familiar to the rapid prototyping industries, contour crafting has the potential to automate the construction of whole composite structures. A building or colony of buildings each with a different design may be constructed in a single run. Embedded in each structure all internal components such as piping, electrical conductors, and reinforcement modules. (302) This technology suggests future potential for the large-scale rapid construction of non-standard, geometrically complex architecture and indeed "fully 3D forms in building scale elements".

Direct rapid manufacturing at building scale is still in its infancy, however it is possible to indirectly manufacture "fully 3D" architectural components using a combination of digital and traditional means. Commercially available Solid Freeform Fabrication (SFF) and CNC manufacturing can be used to rapidly create molds, patterns and tools for fabrication processes such as casting. Although build chambers are currently limited, large and complex components can be subdivided and assembled in sections. Dr. Kevin Rotheroe has initiated research into this method of fabrication in the architectural context. His Freeform Tubes use CNC milled foam as investments for casting non-standard, structural steel components. Although enabling a 
degree of geometric flexibility and demonstrating a promising methodology, Freeform Tubes have not harnessed the full potentials and context specificity afforded by these technologies in order to compliment and enrich contemporary digitally generated architectural forms.

\section{Case Study}

\subsection{Genome Jewelry - integrating digital fabrication}

The Genome jewelry collection demonstrates some of the benefits that can be gained by integrating current and emerging digital technologies with traditional metal 'investment' casting. In particular, the appropriation of computation, design and fabrication strategies across scales and between disciplines.

Elastic membranes exhibit elegant and efficient solutions to spatial and structural design. These 'minimal surfaces' are curiosities that have intrigued mathematicians and designers alike and are recognized for their abilities to rationalize highly complex boundary conditions. The pioneering work of Frei Otto, Sergio Musmeci and more recently that of Ingenhoven Overduik \& Associates, UN Studio and Minifie Nixon Architecture exemplify the use of these surfaces in building design and construction. Genome jewelry and its associated research began as an investigation into the use of digitally generated minimal surfaces as an architectural design tool.

The Surface Evolver written by Professor Kenneth Brakke (Department of Mathematical Sciences, Susquehanna University) is a digital tool that emulates the mathematics and behavior of minimal surfaces. This software enables the generation of unique complex forms and geometries across a variety of scales. Using Surface Evolver alongside a combination of 3D modeling packages, the Genome collection has been meticulously crafted as a comprehensive data set containing all necessary fabrication information. A high-resolution SFF system was used to build the virtual model as a wax object. The 'printed' master wax then translated into precious metals using a common method of investment casting. The industry contacts involved in the process are invigorated by a fresh complexity and the challenge it represents to traditional methods of manufacturing. The information exchange throughout this research has prompted the casting firm Lenrose to purchase the specific high-resolution SFF system used for Genome jewelry. Due to the precision of the casting technique, artifacts from the fabrication process can be seen as contours on the surface of the objects. The fabrication of the final sterling silver and gold pieces has been adjusted to express permutations of surface articulation and patterning that illustrate the numerous potentials, variables and unexpected outcomes implicit to digital design and fabrication strategies.

Digital environments enable the concurrent generation of design and manufacturing information. The complex, intricate and specific geometries explored in the Genome collection were manufactured without the use of 2D information. The final forms, a physical (re)production of precisely crafted 3D data. Beyond the implications of liberating design, digital tools and technologies question the very nature of representation and construction. This research resonates with current and future potentials for the architecture, engineering and construction industries.

Prior to the late nineteenth century, the primary metal used in construction was iron. The flexibility and strength of cast iron freed designers to create new structural forms impossible in stone. Despite its innovation and potential, the tradition of casting metal structural elements with complex geometries has struggled during recent history. The mass-production of rolled and extruded steel from the late nineteenth century has ultimately led to the standardization of construction methods. Twentieth century industry has developed more efficient casting techniques and better-suited construction materials, however the difficulty and primary expenses involved in casting processes have always been the production of tools, patterns and molds. Casting has therefore developed into a specialized craft primarily used for its economy of scale and suitability to mass-production. Rotheroe's research shows that digital fabrication technologies provide a vital framework to alleviate some of these limitations, engaging concepts of mass-customization and enabling the economic manufacture of unique non-standard casting tools.

By appropriating, mastering and integrating digital fabrication with design and manufacturing strategies across scales and between disciplines, Genome jewelry challenges traditional notions of craftsmanship and successfully demonstrates a specific contemporary process with potentials at a variety of scales. The Genome designs debuted in $2^{3} @$ Sherman Galleries Sydney 2006 
and have since been exhibited at the Kunst Museum Germany, $2^{\text {nd }}$ Architecture Biennale Beijing and New Craft Future Voices Conference Scotland.
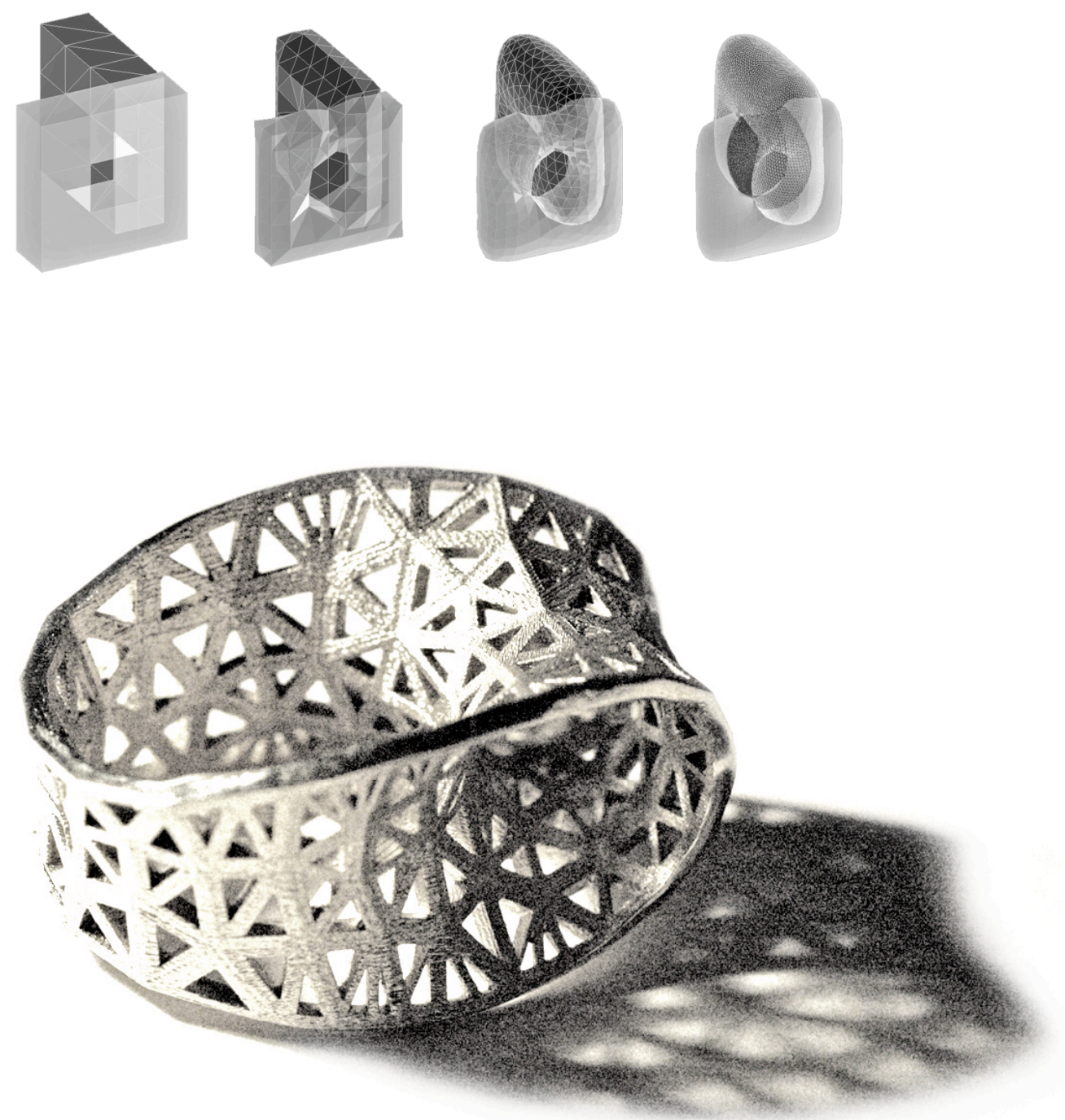

Picture 1: Genome - Not ring 2006

\section{Case Study}

\subsection{Evolutionary Plasticity - context-specific structures in architecture}

When modern man builds large load-bearing structures, he uses dense solids; steel, concrete, glass. When nature does the same, she generally uses cellular materials; wood, bone, coral. There must be good reasons for it.

Prof. M. F. Ashby, University of Cambridge

Nature always achieves its objectives economically, with the minimum energy, conserves its resources and completely recycles its waste.

http://www.daimlerchrysler.com/

The success of the Genome jewelry investigation has prompted further exploration into digital means of design and fabrication at larger scales. Evolutionary Plasticity is being undertaken as research into the suitability of SFF and CNC technologies as indirect manufacturing tools for large-scale components that can be used in the architecture, engineering and construction industries. Specifically this study looks at combining traditional metal casting methods with 
contemporary digital fabrication to enable the economic production of molds, tools and patterns for the indirect manufacture of non-standard, geometrically complex architectural components. It is expected this production methodology will be widely applicable to the architecture and construction industries by facilitating "fully 3D forms in building scale elements." To test this idea, a computational process has been guided to generate optimized, context-specific structural forms that challenge traditional modes of fabrication.

Naturally occurring structures such as trees, bone, coral, sponge, foams and bio-mineralized protist shells exhibit flamboyant geometries that simultaneously negotiate several environmental conditions with minimal energy and material consumption. This negotiation of contextual factors achieves a near uniform stress distribution throughout the structure. The Axiom of Uniform Stress is a phrase coined by theoretical physicist, Professor Claus Mattheck to describe the adaptive growth strategy of naturally occurring self-optimizing structures. Trees have the ability to add material in order to compensate for differentiated environmental stresses. Bones can further restructure material deposits to accommodate temporal changes to their environment. Contemporary computation techniques such as Bi-directional Evolutionary Structural Optimization (BESO), the similar Extended Evolutionary Structural Optimization (EESO) and Soft-Kill Option (SKO) are tools for removing low stress regions and adding material to high stress areas of a 3D digital model under specified loading conditions including consideration of scale, topology and material properties. The BESO algorithm is written by Dr. Xiadong Huang from the Innovative Structures Group at RMIT University and extends the concept of Evolutionary Structural Optimization (ESO) initially presented by Professors Mike Xie and Grant Stevens in 1992 at the International Conference on Computational Engineering Science in Hong Kong. BESO iterates a virtual model toward uniform stress. The framework for the original BESO model is a set of basic parameters distilled from context analysis. These manifest as preliminary geometry, domain scale, load cases, boundary conditions and other design properties such as fixed areas. Adjusting and restructuring material distribution can achieve reduced consumption and increased overall strength-to-weight ratio. This process can be applied at numerous scales and enables the evolution of optimized macrostructures, substructures and microstructures suited to lightweight context-specific building components. The resulting geometries exhibit an elegant combination of function, form and efficiency

The design context for Evolutionary Plasticity is a popular local music venue where an obtuse concrete column interferes with privileged views toward the performance stage. Removal altogether is prohibitively expensive. Replacing it with an optimized porous structure could ease much of the visual interference and provide a functional, intriguing and interactive replacement.

At this point the objects pictured are conceptual, demonstrating three possibilities for engaging the BESO algorithm to generate optimized structural systems. A contextual survey is necessary to finalize the exact computational parameters to ensure the desired design outcomes are attained. Illustrated are potential macro, sub and micro-optimization schemas that can be applied to achieve enhanced strength to weight ratio components that combine a material elegance with functional and structural logic. The macrostructure accomplishes a seamless junction with planar surfaces and illustrates a way of integrating standardized structural systems. The resulting geometry of the substructure optimization acts to enhance rigidity similar to the internal structure of bones. The internal bracing becomes dense in areas of high load transference, adding interest and differentiation to an otherwise smooth form. This optimization schema can be used to create integrated reinforcement and formwork for complex cast composites as it generates hollow sections. The filigree microstructure shows a further level of optimization at the level of surface. For current purposes the structural logic has been appropriated from the deep-sea sponge Euplectella aspergillum also known as the Venus flower basket. This sponge is made of very fine silica material and though inherently brittle, has a particularly robust structure due to its uniquely integrated cross-bracing system. Nature's structural systems demonstrate the principle of lean construction, using minimal energy and material to function optimally. The building and design industries must capitalize on emerging digital technologies that can be integrated to achieve leaner construction methods. Beyond minimizing energy and material consumption, digital tools can be utilized to dramatically streamline the construction process reducing waste, assembly time and on-site labor.

The resulting complexity of evolutionary-based form finding amplifies the need for new and innovative means of fabrication. The level of detail attained in the microstructure optimization is currently only achievable in functioning architectural elements by employing an analogous fabrication process to the one used in the Genome jewelry research. As the examples show, achieving highly optimized architectural componentry is possible by integrating digital technologies with traditional freeform manufacturing processes such as casting. Similar to the 
Genome jewelry, the plaster models pictured have been directly fabricated from a virtual 3D model using the Z-Corp Spectrum, a typical SFF system. The Z-Corp system also supports a proprietary starch material engineered for the investment casting process. It is expected that subdividing the final object will enable a full-scale pattern to be produced via this method. This pattern could then be used as the investment for casting a "fully 3D" building scale component
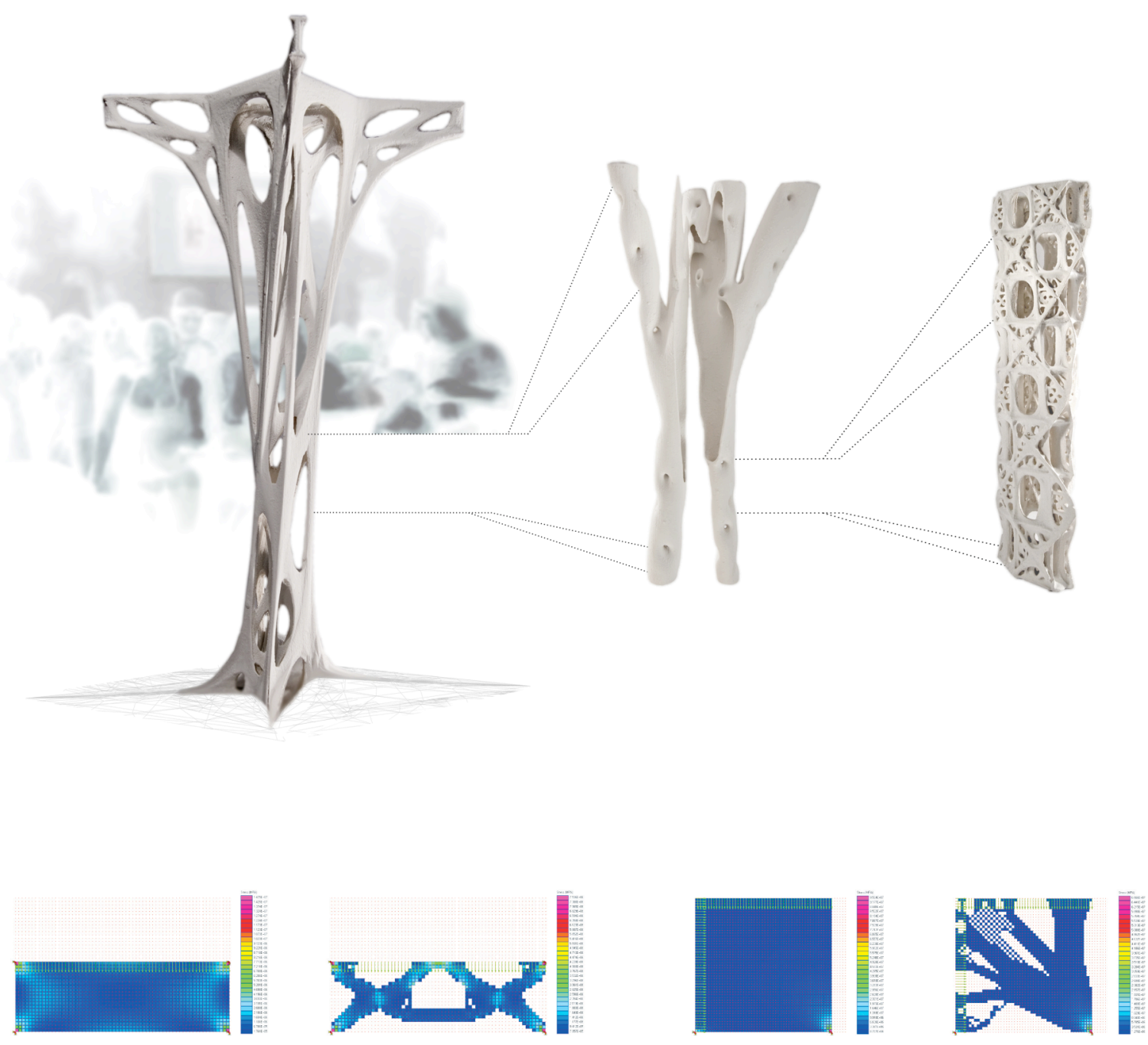

Picture 2: Evolutionary Plasticity - concept models and example diagrams illustrating the removal of low stress regions using Evolutionary Structural Optimization (Xie \& Steven)

The next stage of Evolutionary Plasticity looks at viable methods of integrating specific digital fabrication and metal casting technologies. Of particular interest are investment, sand and 'lost foam' casting. Their differences are marked and range from size and weight limitations to part complexity constraints. The most promising and recent method is 'lost foam', a healthy mix of sand and investment casting. A sacrificial pattern generally made of polystyrene is embedded in sand with feeder channels attached. Molten metal is poured directly into the feeders, vaporizing the foam as it takes shape. This casting method can be used for components up to 50 tons and imposes little limit on form or complexity.

\subsubsection{A note on aesthetics:}

The iterative reduction of irrelevant material using evolutionary-based computational processes returns architecture to its modernist musings. By distilling objects according to functionalist principles are we not effectively practicing modernist theory in an evolutionary fashion? Is it not ironic that the resulting forms are exceptionally flamboyant, challenging traditional methods of fabrication and the industrialist ideology of mass-production? Fortunately and excitingly, the 
actualization of "fully 3D" non-standard, context-specific, optimized strength-to-weight ratio components is a viable prospect in post-industrial digital societies.

\section{Conclusion}

The digital revolution has heavily impacted architectural thought, research and practice. The contemporary vocabulary of digitally based architecture and practice emerged during the midnineties and heralded a new era of forms and form finding. This work challenged the profession, questioning traditional notions of design, manufacturing and construction. Its legacy is fundamental to the development of a new cultural aesthetic. As suggested by Chaszar, it is time to look beyond the surface of architecture to increasingly efficient and context-specific "fully 3D" structural systems that suitably compliment digitally generated architectural forms. The coming generation of architects are increasingly conversant in digital design tools, however a knowledge gap emerges when considering fabrication techniques for complex geometries. The convergence of representation and production information in the digital paradigm signifies a crucial opportunity for architecture. Information-driven modeling enables associative and/or explicit 3-Dimensional models with embedded data sets that can drive computer aided manufacturing tools. As illustrated by the included case studies, active exploration and understanding of available and emerging technologies coupled with an intimate, first-hand knowledge of tools and materials evolves the role of the architect as $2 \mathrm{D}$ documentation providore toward the notion of Master data craftsman, facilitator of the design, fabrication and construction processes.

\section{References}

Chaszar, Andre. "Blurring the Lines: An Exploration of Current Cad/Cam Techniques." Blurring the Lines. Ed. Andre Chaszar. West Sussex: Wiley Academy, 2006.

Khoshnevis, Behrokh. "Mega-Scale Fabrication by Contour Crafting." Industrial and Systems Engineering. 1.3 (2006): 301-20.

Kolarevic, Branko. Architecture in the Digital Age: Design and Manufacturing. Ed. Branko Kolarevic. New York: Taylor \& Francis, 2005.

Kudryavstev, Pyotr. "People in the know: Bernard Franken's craftsmanship” Vedomosti. 2005.

Mattheck, Claus. "Biomechanics.” Forschungszentrum Karlsruhe Institute for Material Research. 2005.

Rotheroe, Kevin. "Manufacturing Freeform Architecture." New Technologies in Architecture Computer-Aided Design and Manufacturing Techniques. Harvard Graduate School of Design: Cambridge, 2000.

Schodek, Daniel L. Digital Design and Manufacturing: Cad/Cam Applications in Architecture and Design. 1 ed. New Jersey: John Wiley \& Sons, 2005.

Xie, Mike Y. and Steven, Grant P. "Shape and layout optimization via an evolutionary procedure." International Conference on Computational Engineering Science. 1992. 471.

Xie Y.M., Huang X., Tang J.W. and Felicetti P. "Recent advances in evolutionary structural optimization." Frontiers of Computational Sciences Symposium. 2005. 13-20.

NOTE: All images appearing in this document are the work of the author. 